\title{
Enhancement of low-quality fetal electrocardiogram based on time-sequenced adaptive filtering
}

\author{
E. Fotiadou ${ }^{1}$ (D) J. O. E. H. van Laar $^{2} \cdot$ S. G. Oei ${ }^{2} \cdot$ R. Vullings ${ }^{1}$
}

Received: 4 April 2018 / Accepted: 10 June 2018 / Published online: 25 June 2018

(C) The Author(s) 2018

\begin{abstract}
Extraction of a clean fetal electrocardiogram (ECG) from non-invasive abdominal recordings is one of the biggest challenges in fetal monitoring. An ECG allows for the interpretation of the electrical heart activity beyond the heart rate and heart rate variability. However, the low signal quality of the fetal ECG hinders the morphological analysis of its waveform in clinical practice. The time-sequenced adaptive filter has been proposed for performing optimal time-varying filtering of non-stationary signals having a recurring statistical character. In our study, the time-sequenced adaptive filter is applied to enhance the quality of multichannel fetal ECG after the maternal ECG is removed. To improve the performance of the filter in cases of low signal-to-noise ratio (SNR), we enhance the ECG reference signals by averaging consecutive ECG complexes. The performance of the proposed augmented time-sequenced adaptive filter is evaluated in both synthetic and real data from PhysioNet. This evaluation shows that the suggested algorithm clearly outperforms other ECG enhancement methods, in terms of uncovering the ECG waveform, even in cases with very low SNR. With the presented method, quality of the fetal ECG morphology can be enhanced to the extent that the ECG might be fit for use in clinical diagnostics.
\end{abstract}

Keywords Electrocardiography $\cdot$ Fetal ECG de-noising $\cdot$ Fetal ECG enhancement $\cdot$ Time-sequenced adaptive filter

\section{Introduction}

The fetal electrocardiogram (ECG) can be used to monitor the fetal condition from early pregnancy until delivery [24]. Analysis of its waveform provides information that can assist clinicians in making more appropriate and timely decisions during labor [17]. This can be applicable in the case of fetal hypoxia that occurs as a result of oxygen deprivation of the fetus during parturition. The condition is often accompanied by acidosis and is life-threatening unless prompt interventions are undertaken to restore well-oxygenated blood to fetus. Fetal hypoxia is found to be associated with changes in the ECG waveform [33]. The recording of the fetal ECG can be carried out by an invasive electrode or by placing skin electrodes on the maternal abdomen. Unfortunately, in abdominal

E. Fotiadou e.fotiadou@tue.nl

1 Department of Electrical Engineering, Eindhoven University of Technology, 5612 AP Eindhoven, Netherlands

2 Department of Obstetrics and Gynaecology, Máxima Medical Center, 5504 DB Veldhoven, Netherlands recordings, the obtained signals are substantially contaminated by interferences and noise that vary depending on the gestational age, position of electrodes, skin impedance, etc. [24]. The main sources of interference include the maternal ECG, maternal and fetal muscle noise, powerline interference, baseline wander, movement artifacts, and multiple layers of dielectric biological tissues through which the electrical signals must pass. The signals from some of these interferences overlap in both time and frequency with the fetal ECG, complicating the extraction of the fetal ECG through conventional filtering techniques.

Despite the difficulties in acquiring fetal ECG signals non-invasively, a number of different techniques have been proposed in the literature such as neural networks [7, 21], wavelet-based methods $[18,23]$, singular value decomposition [22], blind source separation [6, 26, 42, 43], adaptive filtering $[1,14,27,39,40]$, as well as combinations of different algorithms [15, 38, 41]. Clifford et al. [9] review the key achievements and the follow-up research generated as a result of the PhysioNet/Computing in Cardiology Challenge 2013 [35]. The challenge focused on fetal heart rate estimation and QT measurement in an automated way and managed to accelerate algorithm development in these areas $[3,5,30,36]$. 
However, the extracted fetal ECG signal usually has a low signal-to-noise ratio (SNR) and additional processing is required to further enhance its quality. Beat-to-beat averaging [25] can be employed to improve the SNR of the signal. This approach, however, has the disadvantage that individual variations in pulse shape can be lost. In [39], an adaptive Kalman filter is developed that varies the number of complexes to be averaged according to the characteristics of the ECG signal. The filter is able to infer whether the ECG signal is corrupted by noise or dynamic variations and adapt the number of averages accordingly, in that way preserving the pulse variations. However, the filter is not extensively evaluated in fetal ECG signals with low SNR.

The proposed method focuses on the post-processing of the extracted fetal ECG signals, for enhancing their quality, based on adaptive filtering. Adaptive filters [11, $31,40]$ have the ability to adjust their parameters autonomously and have been widely used to remove uncorrelated noise components when the noise characteristics are a priori unknown. Since the fetal ECG is a time-varying signal, adaptive filtering seems appropriate for estimating the fetal ECG. However, a least mean squares (LMS) adaptive filter [13] has been proven inadequate to fulfill this role due to the low SNR, complexity, and non-stationarity of the fetal ECG. Despite the substantial background noise removal achieved by the LMS adaptive filter, the signal distortion was too severe, causing relevant ECG morphology to disappear.

The fetal ECG signal is highly non-stationary, and a LMS filter is typically unable to track these rapidly varying non-stationarities. In [12], the time-sequenced adaptive filter (TSAF) has been suggested for the estimation of a class of non-stationary signals having a recurring statistical character and has shown good performance for detecting fetal ECG $[1,14]$. The TSAF can be conceptualized as a bank of LMS adaptive signal enhancers that can achieve a rapidly varying impulse response. Fetal pulses differ among each other but have similar statistical properties. When the pulses are aligned according to a fiducial point, the statistics can be computed over the ensemble of pulses. The fiducial point at which the statistical properties of the signal renew is called the regeneration time. Each adaptive signal enhancer becomes an expert at filtering a specific signal segment between the regeneration times. The advantage of the method is that it does not require a priori knowledge of signal characteristics. However, an external input is needed to determine the regeneration times. In $[1,14]$, the $\mathrm{R}$ peak locations were detected to determine the regeneration times; Ferrara and Widrow [14] used a matched filter followed by peak detection, whereas Adam and Shavit [1] used a synchronized Doppler ultrasound signal.
The results of applying the TSAF to fetal ECG enhancement, presented in $[1,14]$, show that, in case of poor SNR, the characteristic waves of the ECG cannot be distinguished after filtering. Moreover, as mentioned in [1], when the input SNR is relatively low, the effect of the filter's regeneration time can be seen in some cardiac cycles. This paper presents an improvement of the TSAF for fetal ECG enhancement and demonstrates the feasibility of time-sequenced adaptive filtering for enhancing the quality of the fetal ECG to the extent that the morphology of the signal is preserved. This improvement involves the enhancement of the reference inputs prior to their use in the TSAF, yielding an increase in the SNR of the TSAF output, and the elimination of aforementioned effects of the regeneration time via the use of overlapping filters.

\section{Materials and methods}

\subsection{The time-sequenced adaptive filter}

The TSAF provides a separate LMS enhancer for each signal sample in an ECG cycle, as opposed to the original adaptive signal enhancer [11] that consists of a single LMS filter. Thus, there are a number of adaptive filters equal to the length of an ECG complex and each one is updated in every ECG cycle. The structure of the filter is depicted in Fig. 1. An external input, known as the sequence number, determines which LMS enhancer to be used at each point in time. The LMS adaptive filter is a stochastic gradient-based algorithm that utilizes the gradient vector of the filter tap weights to converge to the optimal Wiener solution [40]. Each

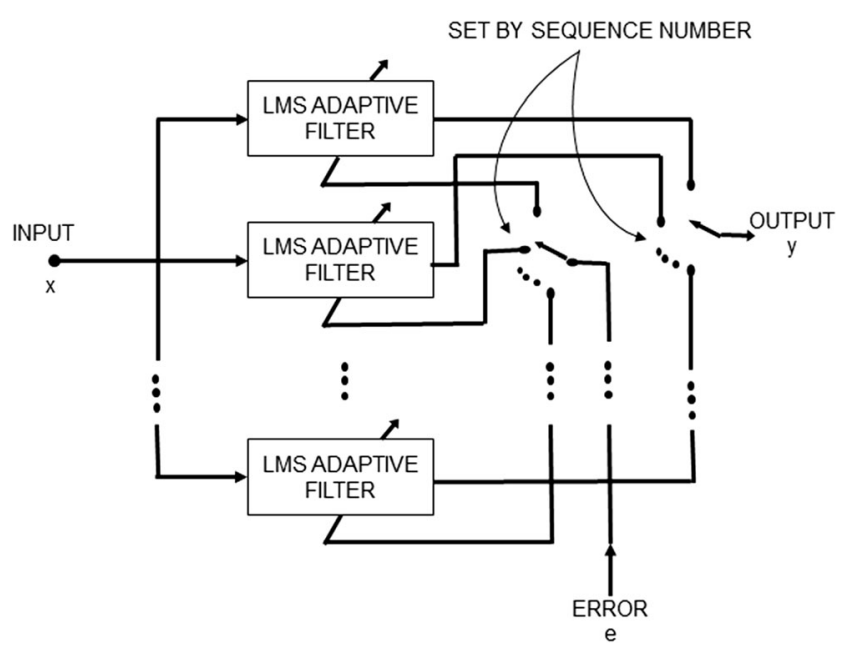

Fig. 1 Structure of the time-sequenced adaptive filter. The filter is realized as a bank of LMS adaptive filters. The sequence number determines which LMS filter is used at each point in time 
iteration of the LMS algorithm consists of the following steps:

1. Calculation of the filter output

$y(n)=\mathbf{w}^{T}(n) \mathbf{x}(n)$

where $\mathbf{x}(n)$ is the filter input and $\mathbf{w}(n)$ is the filter weights. The size of the vectors is equal to the filter order $(P)$.

2. Estimation of the error signal which is needed to update the filter coefficients in the next step

$e(n)=d(n)-y(n)$

where $d(n)$ corresponds to the $n$th sample of the desired solution (in our case, the signal to be enhanced; see Fig. 2).

3. Update of the filter weights for the next iteration

$\mathbf{w}(n+1)=\mathbf{w}(n)+\mu e(n) \mathbf{x}(n)$

where $\mu$ is a step-size parameter.

The weights $(\mathbf{w}(n))$ are initialized as zero. The step-size parameter controls the rate at which the weights change. The choice of this parameter is critical for balancing the convergence of the filter versus its stability. Selecting a step size that guarantees stability and ensures fast enough convergence is difficult due to the sensitivity of the LMS algorithm to scaling of its input [11]. To resolve this issue, in this work, we use the normalized least mean squares (NLMS) filter instead of the LMS. NLMS is an extension of the standard LMS algorithm with the difference that it uses a time-varying step size, yielding a faster convergence as opposed to the LMS algorithm [10]. The step size used by the NLMS filter is defined as:

$\mu^{\prime}(n)=\frac{\bar{\mu}}{\mathbf{x}(n)^{T} \mathbf{x}(n)}$

where $\bar{\mu}$ is a scalar which allows for a change in the adaptation speed. The normalization of the step size with the power of the input signal $(\mathbf{x}(n))$ makes the algorithm insensitive to scaling.

\subsection{Proposed method}

\subsubsection{Enhancement of reference signals}

In this study, we have performed pre-processing and maternal ECG removal following the method developed by Varanini et al. [36]. The residual signals comprise of fetal ECG and a significant amount of remaining noise. Typically, the fetal ECG components among different channels are correlated, whereas some remaining noise components such as muscle noise are mostly uncorrelated. We propose the use of an augmented TSAF (aTSAF) to enhance the quality of multichannel fetal ECG by attenuating the uncorrelated noise (Fig. 2). The channel to be de-noised is considered as the primary channel and the other channel(s) as the reference channel(s). In Fig. 2, the use of channel 1 as primary channel is just an example. Every channel that we wish to de-noise can be considered as
Fig. 2 Block diagram of the proposed $N$-channel timesequenced fetal ECG enhancement method. The reference channels (no. 2-no. $N$ ) are enhanced prior to filtering. The filter output is an estimate of the de-noised fetal ECG signal of channel 1

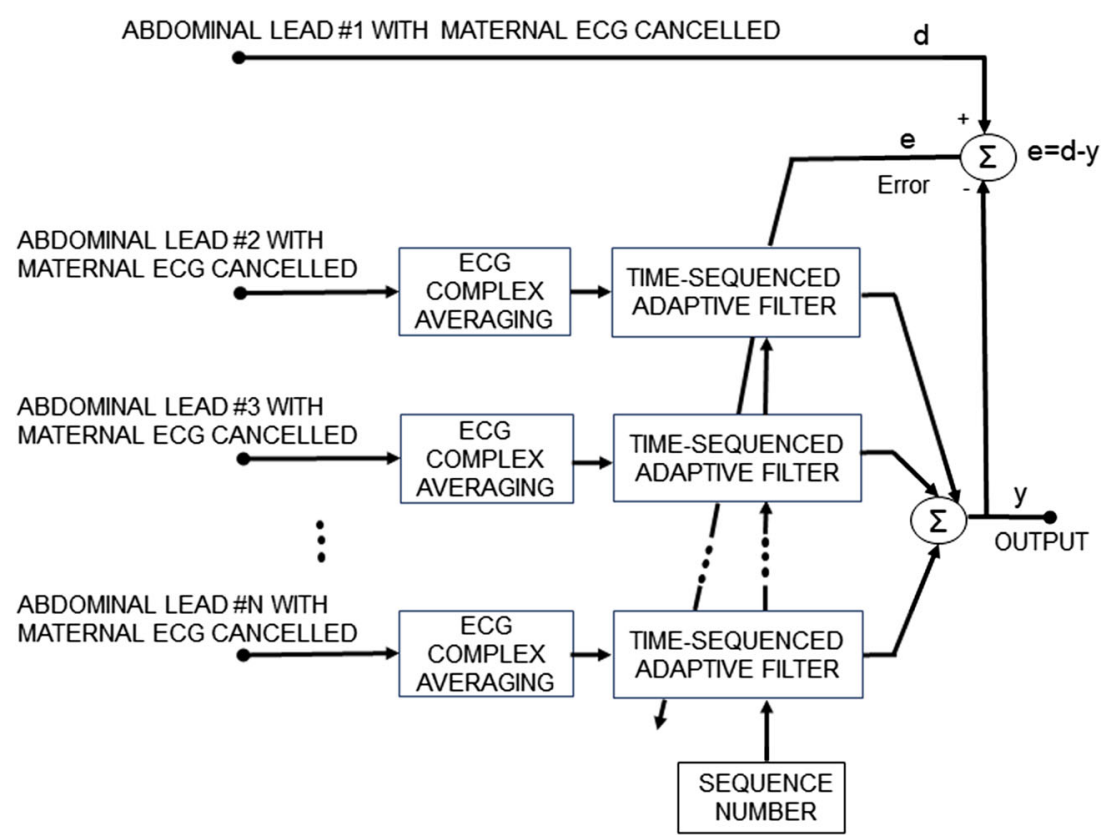


primary channel. The reference channels should be correlated with the primary one and should have sufficiently high SNR to yield satisfactory performance of the TSAF. To ensure an acceptable SNR of the reference inputs, in this work, we enhance the quality of the reference channels by ensemble averaging, prior to using them in the TSAF. The ensemble averaging includes the detection of the $\mathrm{R}$ peak locations by the method described in [36], the alignment of successive complexes according to these locations, and the ensemble averaging of aligned ECG complexes. In the ensemble averaging, we used the ECG of 30 consecutive heartbeats to preserve clinically relevant variations in the ECG and, at the same time, produce substantial enhancement of the ECG [33].

\subsubsection{Scheme for faster algorithm convergence}

After the reference channels have been enhanced, the TSAF combines them via a dynamic linear combination, where the weights in this linear combination are optimized for minimizing the error between the TSAF output and the primary input. To maintain a high rate of convergence, without risking instability of the process, a scheme is employed as described in the work of Cano et al. [8]. According to it, the filter weights $(\mathbf{w}(n))$ are updated not just in the prior ECG cycle but also in the current cycle where they are used for filtering. The scheme makes the assumption that immediate neighboring data samples are highly correlated and can be used to approximate each other; thus, the weights can be updated in the cycle being filtered. It is only employed for a given number of ECG cycles to assist the algorithm to achieve faster convergence.

\subsubsection{Reduction of the regeneration time effect}

The TSAF requires the knowledge of the location of the fetal ECG fiducial points in order to determine the regeneration times. The sequence number at each regeneration time is (re) set to 1 and increases by 1 for each data sample. Since the fetal PR interval is approximately $100 \mathrm{~ms}$ [37], the regeneration times are chosen to occur $160 \mathrm{~ms}$ before the detected $\mathrm{R}$ peaks. In such a way, the start of the sequence occurs before the $\mathrm{P}$ wave starts. The sequence length defines how many adaptive signal enhancers constitute the TSAF. Ideally, we want the sequence length to be equal to the ECG complex length but this varies from cycle to cycle. Hence, we defined the sequence length to be $110 \%$ of the mean interval between the fetal $\mathrm{R}$ peaks. In this way, it is ensured that in most cases, the sequence length is bigger than the length of the ECG cycle, allowing all the samples in the cycle to be processed by a separate adaptive filter. This implies, however, that in most cases, there are overlapping signal parts that are filtered twice. These parts are smoothed by averaging the contributions of both overlapping complexes. To be more specific, the contribution of the first ECG complex is gradually reduced while the contribution of the second complex is gradually increased. Inevitably, there are cases where the length of the sequence is smaller than the size of the ECG complex. In this situation, gaps exist between successive complexes. The samples in these gaps are filtered by the same LMS adaptive filter that was used for the last sample in the sequence. The weights of this filter are updated only once in the current cycle. This guarantees that all the filters in a cycle converge with similar speed. By selecting the start and length of the sequence in the way described before, we avoid that the characteristic waves of the ECG complex fall outside the borders of the sequence in cases of ECG complexes longer than the sequence length. This is very important since the waves contain all the useful information about the physiological state of the fetus. Following the described approach, the regeneration time effect noticed in [1] can be significantly reduced.

\subsection{Data description}

The fetal ECG signals, even after the maternal ECG has been removed, are still affected by noise. It is hence impossible to have a gold reference (i.e., clean signal) that can be used to quantitatively validate the proposed algorithm. As a surrogate, in this study, the proposed method is extensively validated based on simulated signals of the Fetal ECG Synthetic Database (FECGSYNDB) of PhysioNet $[2,16]$. To illustrate the potential of our method on real data, we have included results obtained from data in the Abdominal and Direct Fetal Electrocardiogram Database of PhysioNet [20].

The FECGSYNDB consists of 1750 synthetic abdominal signals with 34 channels, sampling frequency of $250 \mathrm{~Hz}$, and duration of $5 \mathrm{~min}$. The database includes ten simulated pregnancies with seven different physiological events as shown in Table 1. The signal-to-noise ratio of the simulated signals varies from 0 to $12 \mathrm{~dB}$ in steps of $3 \mathrm{~dB}$. In each simulation, signals are generated five times for statistical purposes. In this work, we use six channels (i.e., 1, 8, 11, 22, 25, and 32) for evaluation of our algorithm, as suggested by Andreotti et al. [2]. The signals simulating twin pregnancy (fifth case, Table 1) are excluded from our analysis, since the proposed algorithm is not developed to handle this case.

The Abdominal and Direct Fetal Electrocardiogram Database contains multichannel fetal electrocardiogram (FECG) recordings obtained from five different women in labor, between 38 and 41 weeks of gestation. Each recording contains four signals acquired from the maternal abdomen and one scalp ECG signal. The recordings have a duration of $5 \mathrm{~min}$ and are sampled at $1000 \mathrm{~Hz}$.

The signals of both databases are pre-processed before the proposed method is applied to them. First, the signals are resampled to $500 \mathrm{~Hz}$ to have a common reference. Then, the open-source algorithm of Varanini et al. [36] is applied to the signals. According to this algorithm, first, the baseline wander 
Table 1 Description of the seven cases of physiological events of the synthetic signals of the Fetal ECG Synthetic Database

\begin{tabular}{ll}
\hline Case & Description \\
\hline Baseline & Abdominal mixture (no noise or events) \\
Case 0 & Baseline (no events) + noise \\
Case 1 & Fetal movement + noise \\
Case 2 & Acceleration or deceleration of maternal \\
& and fetal heart rate + noise \\
Case 3 & Uterine contraction + noise \\
Case 4 & Ectopic beats for both fetus and mother + noise \\
Case 5 & Twin pregnancy + noise \\
\hline
\end{tabular}

and the powerline interference are removed. After that, the maternal ECG is estimated through independent component analysis (ICA) and singular value decomposition and subsequently subtracted from the signals. Finally, a second ICA is employed to enhance the fetal ECG signal and two QRS detectors are applied in forward and backward directions to obtain the R peak locations. Figures 3 and 4 show an example of simulated data and real data respectively together with the result of the aforementioned pre-processing. In both cases, the extracted fetal ECG after the maternal ECG removal contains a significant amount of remaining noise.

\subsection{Performance measure}

The performance of the method is assessed based on the SNR improvement in the filter output when compared to the input signal. The metric is computed as follows:

$$
\mathrm{SNR}_{\text {imp }}[d B]=10 \log _{10} \frac{\sum_{n=1}^{M}|d[n]-z[n]|^{2}}{\sum_{n=1}^{M}|y[n]-z[n]|^{2}}
$$

where $z[n]$ denotes the original clean fetal ECG signal, $d[n]$ the noisy signal, $y[n]$ the enhanced one, and $M$ the length of the signals. The metric is measured for each channel and subsequently summed over all ECG channels and over all signals.

The proposed method is compared to several other ECG enhancement algorithms. The first one is a wavelet de-noising method that decomposes the signal, thresholds the detail coefficients, and reconstructs the signal to obtain its enhanced version. The 6-order Daubechies wavelet is selected as a mother wavelet because of its similarity to an actual ECG. A fixed threshold is used which is estimated by the minimax principle [34]. Secondly, our algorithm is compared to the ensemble averaging over 30 consecutive ECG complexes. The next reference method is the adaptive Kalman filter described in [39], where the number of the ECG complexes to be averaged is adapted according to the signal characteristics. An additional comparison is made with the multichannel NLMS adaptive signal enhancer [11] and with the NLMS adaptive signal enhancer for which the reference channels are enhanced via our suggested averaging method (we will refer to this method as augmented NLMS, aNLMS), as described in Section 2.2.1. Finally, our method is also compared to the TSAF without pre-processing of the reference channels but including compensation for regeneration time effects.

\section{Results}

\subsection{Parameter optimization}

The proposed algorithm has few parameters that must be chosen: the parameter $\bar{\mu}$ that is used in the calculation of the step size of NLMS algorithm and $P$, the length of the adaptive filters. The value of $\bar{\mu}$ is critical for the algorithm's performance since a wrong choice will restrain the algorithm from convergence. However, once chosen appropriately, this parameter does not need further readjustments because of the scaling tolerance of the NLMS algorithm. The choice for $P$ is less critical.
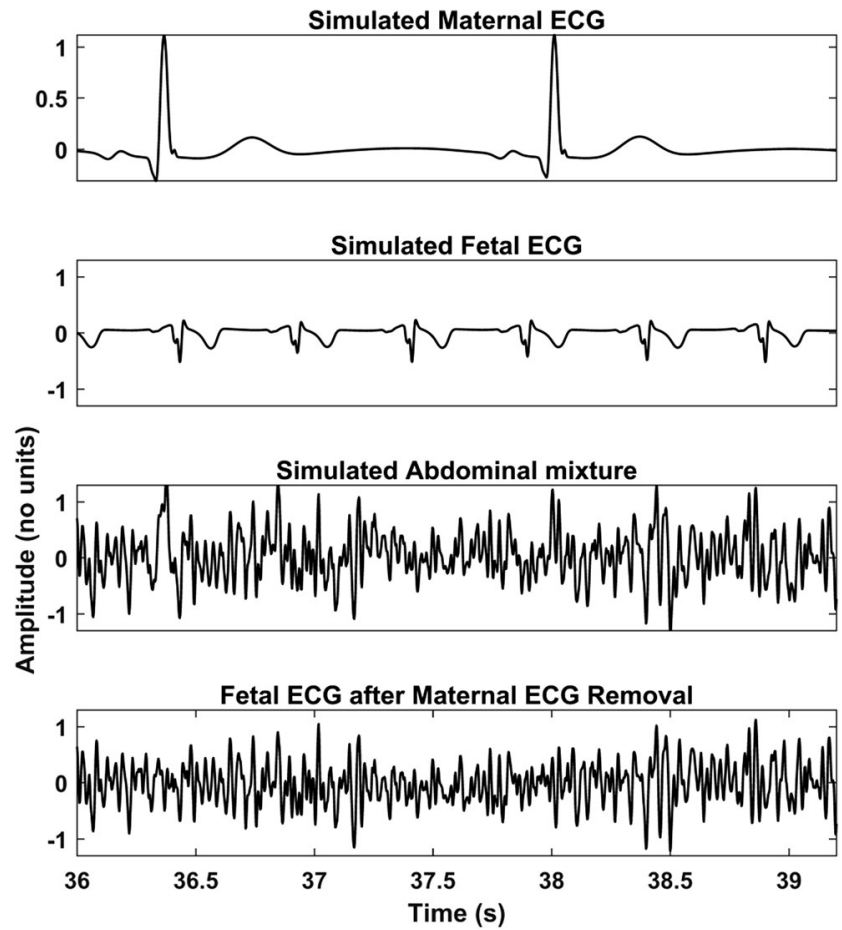

Fig. 3 An example of simulated signals of the FECGSYNDB. The first row shows the simulated maternal ECG; the second the simulated fetal ECG; the third the abdominal mixture, where noise is also added; and the last the extracted fetal ECG after the method of Varanini et al. [36] is applied. The displayed segment corresponds to the third channel (third of the six channels in use) of the tenth simulated pregnancy, SNR 0 , case 0 , and second repetition 

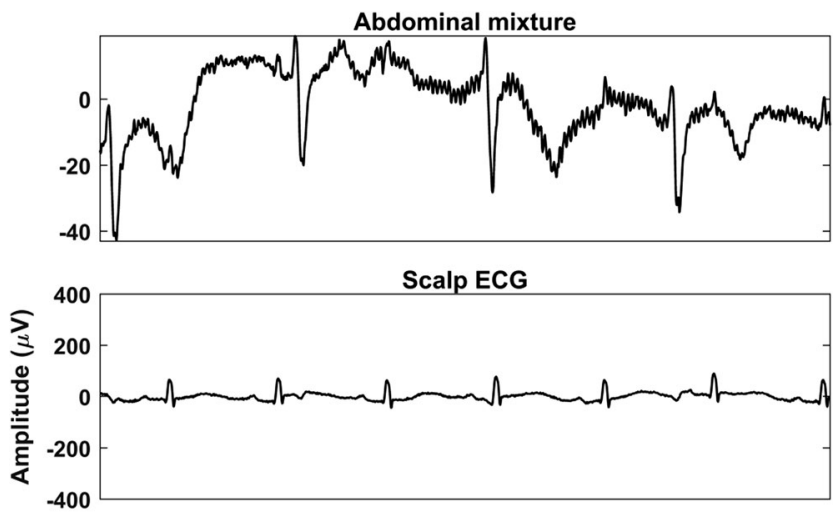

Fetal ECG after Maternal ECG Removal

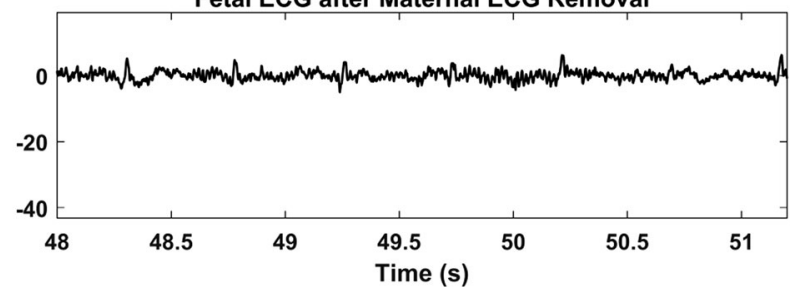

Fig. 4 An example of real signals of the Abdominal and Direct Fetal Electrocardiogram Database. The first row shows a segment of the abdominal mixture of channel 1 for recording "r07." The second displays the simultaneously recorded scalp fetal ECG, while the last the extracted fetal ECG after the method of Varanini et al. [36] is applied

In order to select these two parameters, the simulated signals of the FECGSYNDB are separated in a training and a test set. The first five simulated pregnancies are used as training data while the last five as test. The training set is used to optimize the parameter values, while the test set is used to evaluate the performance of the algorithm. An iterative procedure is performed over several parameter values, and the ones that produce the highest SNR improvement in the training set are finally selected. For $\bar{\mu}$, a search is performed between the values 0.0005 and 0.1 with eight logarithmic steps. Regarding the filter length, the values of 50 and 100 to 800 with steps of 100 are used. For TSAF, NLMS, and aNLMS, the parameter values are selected similarly. The parameter values that produce the best results for all the algorithms are 0.005 for $\bar{\mu}$ and 100 for $P$.

\subsection{Evaluation on the Fetal ECG Synthetic Database}

Our method is evaluated on data from the FECGSYNDB described in Section 2.3. Since the proposed algorithm requires the knowledge of the fetal $\mathrm{R}$ peaks, only the signals for which at least $80 \%$ of the fetal $\mathrm{R}$ peaks are detected within an error of $50 \mathrm{~ms}$ from the actual R peak [4] are included in the evaluation of the algorithm. Table 2 shows the number of signals with successful $\mathrm{R}$ peak detection for each of the different cases of the FECGSYNDB. The numbers correspond to the simulated pregnancies 6-10. As expected in the baseline case, where no noise is added to the abdominal mixture, the
Table 2 The number of the signals with detection for each of the different cases of the FECGSYNDB (125 synthetic signals per case of the test-simulated pregnancies 6-10) successful R peak

\begin{tabular}{ll}
\hline Case & Number of signals \\
\hline Baseline & 121 \\
Case 0 & 94 \\
Case 1 & 106 \\
Case 2 & 84 \\
Case 3 & 78 \\
Case 4 & 30 \\
Total & 513 \\
\hline
\end{tabular}

extracted fetal ECG signals are of relatively good quality and the $\mathrm{R}$ peak detection succeeds in almost all the cases. Case 4 , where ectopic beats are simulated, is the most challenging one and the R peak detection succeeds only in 30 out of 125 occurrences.

Figure 5 illustrates the performance of the proposed method in comparison to the de-noising algorithms mentioned in Section 2.4 for the different SNR values of the input signals. The input SNR refers to the SNR of the signals after the maternal ECG has been removed, and this SNR is averaged over all channels and measurements. From Fig. 5, it can be observed that, for very low SNR values of the input signals, the proposed algorithm clearly outperforms the other algorithms. For input SNR less than $-15 \mathrm{~dB}$, the suggested method provides an additional SNR improvement of at least $3 \mathrm{~dB}$ over the other best-performing methods. For high SNR values (more than $0 \mathrm{~dB}$ ), the conventional TSAF algorithm produces a similar result with our algorithm since there is no need to filter the reference channels. It is worth observing the increase in performance of the aNLMS algorithm over the conventional NLMS. Without our proposed pre-processing of reference signals, NLMS performs significantly worse. From Fig. 5, it can be seen that the averaging of ECG complexes produces good results when the SNR is higher than -10 . However, for lower SNR values of the input signals, the SNR improvement is significantly lower than that of the proposed algorithm. Apparently, different ECG segments should be considered separately to truly enhance the ECG. Plain averaging works on the entire ECG complex and treats it as a whole. On the other hand, our method uses different filtering schemes for different parts of the ECG.

Figure 6 depicts the performance of the suggested method for each of the different cases separately and for all the ranges of available input SNRs. The input SNR values are clustered in six groups of values from -32 to $8 \mathrm{~dB}$ in steps of eight. Input signals with SNR from all these groups are not available for every case, since the SNR is measured after the maternal ECG is removed from the simulated signals. As we can see in Fig. 6, the efficiency of aTSAF for each specific input SNR is similar for the different cases apart from case 4 and the baseline case. Case 4 , as mentioned before, is a challenging case that simulates the presence of ectopic beats. In this case, 


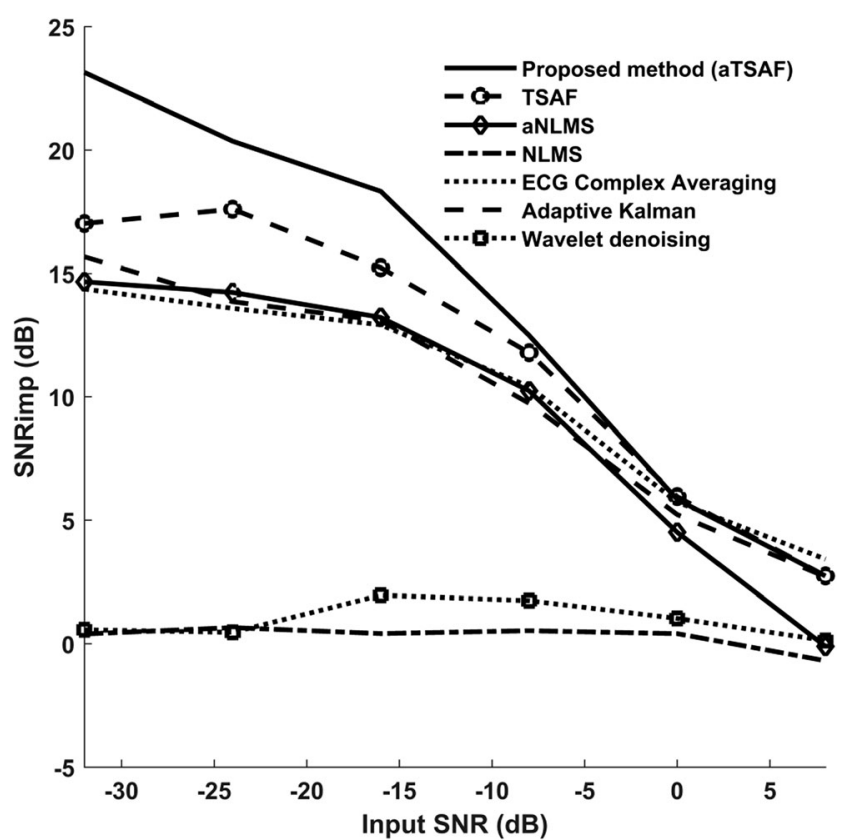

Fig. 5 Performance of different fetal ECG enhancement algorithms in comparison with the proposed algorithm by means of improvement in the SNR of the output when compared to the SNR of the input signal. The results correspond to the average over all channels and cases for the synthetic signals of the FECGSYNDB (simulated pregnancies 6-10)

abrupt changes in the morphology of the ECG signals occur, for a short amount of time, making it relatively impossible for the algorithm to adapt fast enough. In this case, our algorithm adapts to regular heartbeats but is incapable of tracking the sudden changes. Regarding the baseline case, less improvement in SNR is achieved compared to the other cases. The

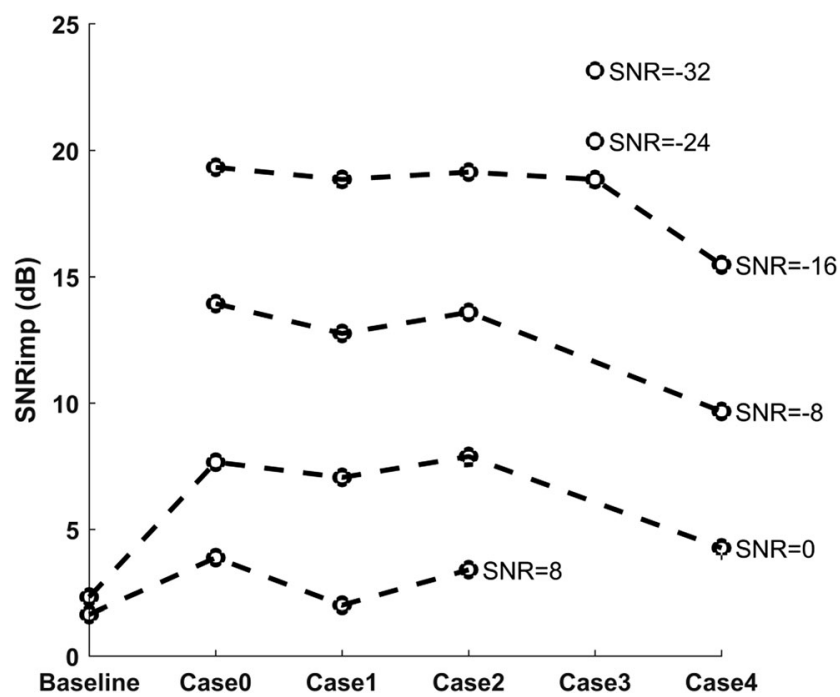

Fig. 6 Performance of the proposed aTSAF algorithm for each of the different cases and the input SNR of the synthetic signals of the FECGSYNDB. The input SNR corresponds to the average of all channels and after the maternal ECG is removed. The "o" circles show the performance only for the cases where there are available input signals with the specific SNR ( -32 to $8 \mathrm{~dB}$ ) reason is that this case does not contain added noise that can be removed by the aTSAF.

The results of processing the simulated signals, which are described in Fig. 3, by our aTSAF method are illustrated in Fig. 7. For the depicted recording, the average SNR of the six channels after the maternal ECG has been removed is $-18 \mathrm{~dB}$. The figure demonstrates that the proposed aTSAF method is capable of suppressing the noise to the extent that the individual waves in the ECG complex become distinguishable. Moreover, there is virtually no distortion of the ECG signal. In this particular case, the suggested algorithm clearly outperforms the other methods. The additional step of enhancing the quality of reference channels - which is the main contribution of this study - makes a significant difference in the performance of the TSAF algorithm, especially for cases with low-quality signals. For the other algorithms, the ECG complex averaging and the aNLMS produce the best results but show still a significant amount of noise in the filtered signals. For the other methods, the morphological information of the ECG cannot be seen.

\subsection{Evaluation on the Abdominal and Direct Fetal ECG Database}

In this subsection, the performance of the same algorithms evaluated in Section 3.2 is evaluated on actual data from the Abdominal and Direct Fetal ECG Database. Concerning the algorithm parameters, the same parameters that were optimized in the FECGSYNDB are used. In the real data, because of the lack of ground-truth data, the performance of the various methods is evaluated qualitatively, as opposed to quantitatively for the simulated data. To demonstrate the potential of abdominal ECG recordings, we have shown the scalp ECG, after high-pass filtering for baseline wander removal, as well in our figures (see, e.g., Fig 8). It should be noted that the scalp ECG is a different ECG lead than the abdominal leads and that they should not be identical, even in case of perfect enhancement. Nevertheless, the individual ECG segments should coincide between abdominal leads and scalp lead.

Figure 8 illustrates the results of various algorithms for the fetal ECG enhancement of channel 1 of recording "r07," which was already depicted in Fig. 4. As seen in Fig. 8, the ECG signal filtered by the proposed aTSAF algorithm is relatively free from noise and the individual waves correspond well to those in the scalp ECG. Pre-processing of the reference channels appears to have a substantial contribution to the performance of both the TSAF and NLMS algorithms, making even the small waves distinguishable. Without our proposed pre-processing, these characteristic waves are often not visible, either because they were suppressed by the filter or because the noise was not suppressed enough.

Figure 9 demonstrates the results of the processing of the four other recordings of the Abdominal and Direct Fetal ECG 

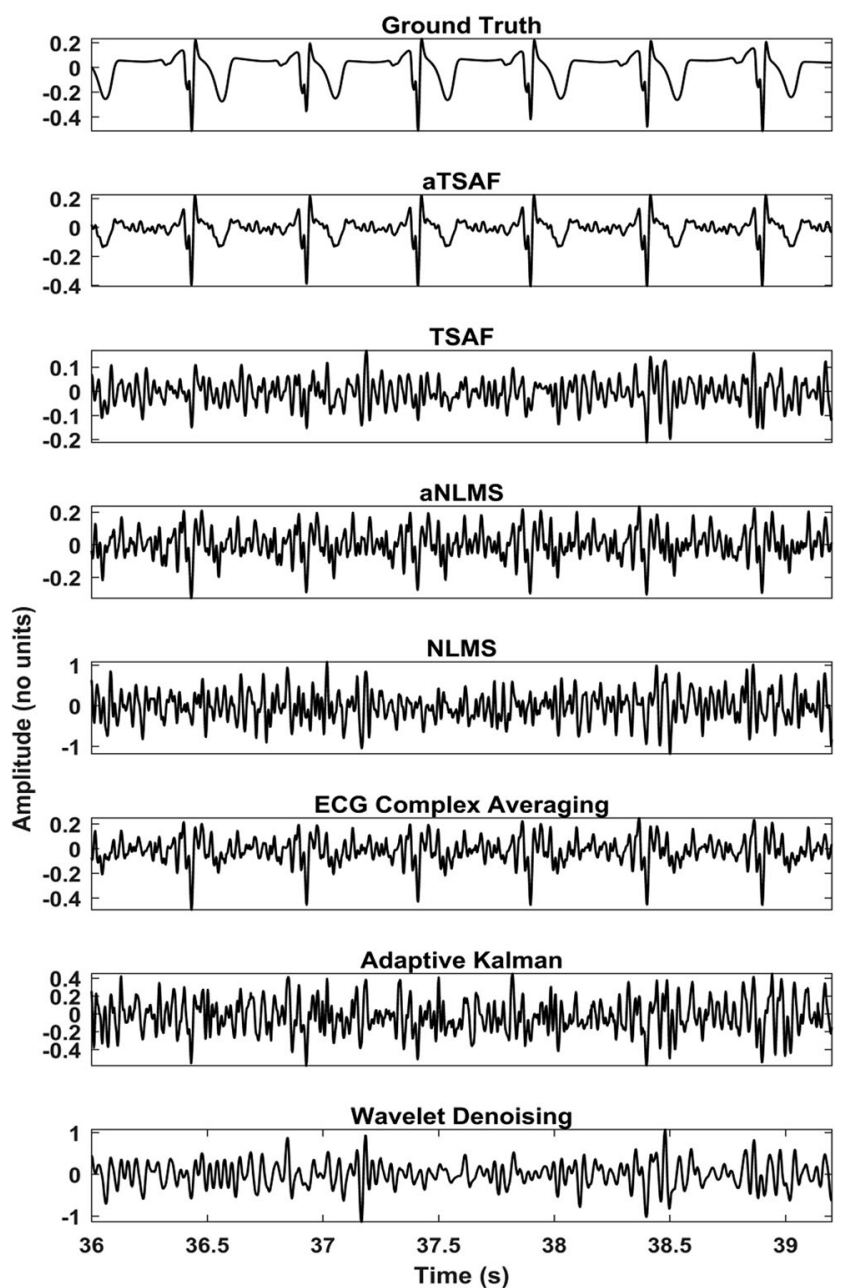

Fig. 7 Enhancement result of the different algorithms for the simulated signals described in Fig. 4. In the first row, the simulated clean fetal ECG signal is displayed for comparison. Only the proposed method (aTSAF) provides a high-quality result in this challenging case of low-SNR input signals $(-18 \mathrm{~dB}$ on average)

Database with the proposed algorithm. The corresponding scalp ECGs are presented together with the results for channel 1 of each recording. In all cases, the suggested method produces a relatively clean ECG signal with morphology that corresponds relatively well to that of the scalp ECG, especially for the P wave and QRS complex.

\section{Discussion}

A method is presented here for post-processing of the extracted fetal ECG after the maternal ECG is removed. In most cases, the extracted fetal ECG still contains a substantial amount of noise that impedes the interpretation of the morphology of the ECG signal by clinicians. Usually, the QRS complex can be detected without further processing of the fetal ECG, due to the high amplitude of the R peak. However, the smaller waves, like the $\mathrm{P}$ and $\mathrm{T}$ waves, can often
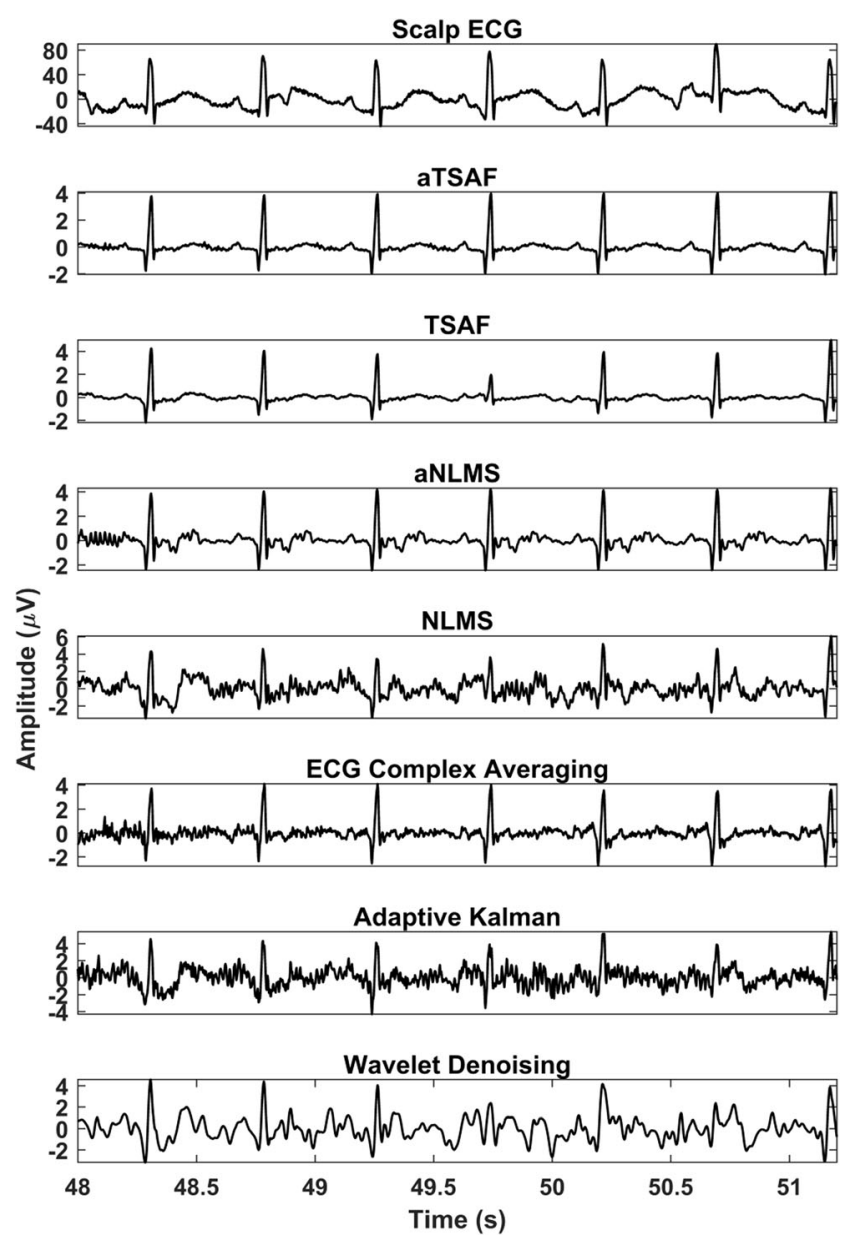

Fig. 8 Comparison of the enhancement results of the different algorithms for channel 1 of recording "r07" of the Abdominal and Direct Fetal Electrocardiogram Database. In the first row, the simultaneously recorded scalp ECG is presented. The noisy signal is shown in Fig. 5. In the output of the proposed method (aTSAF), the characteristic ECG waves are clearly visible and correspond well to those of the scalp ECG

not be readily distinguished. Thus, post-processing of the extracted fetal ECG signal, to enhance its quality, becomes of paramount importance. For this purpose, in the proposed method, the TSAF is improved by increasing the quality of the reference channels and by exploiting overlapping filters to minimize the effect of regeneration times. The improved filter is found to be effective in reducing major components of noise. But more than that, the main contribution of the filter is that after the filtering, the signal morphology is retained to the extent that even the small signal waves can be visually distinguished. As a plus, the method is relatively insensitive to the choice of parameters and, as such, is rather generally applicable.

A limitation of the method is that an estimate of the $\mathrm{R}$ peak locations is required to determine the regeneration times of the filter. However, this does not necessarily impede the use of our filter. First of all, a lot of valuable work has been already done in the area of fetal $\mathrm{R}$ peak estimation $[3,5,30,32,36]$ with 

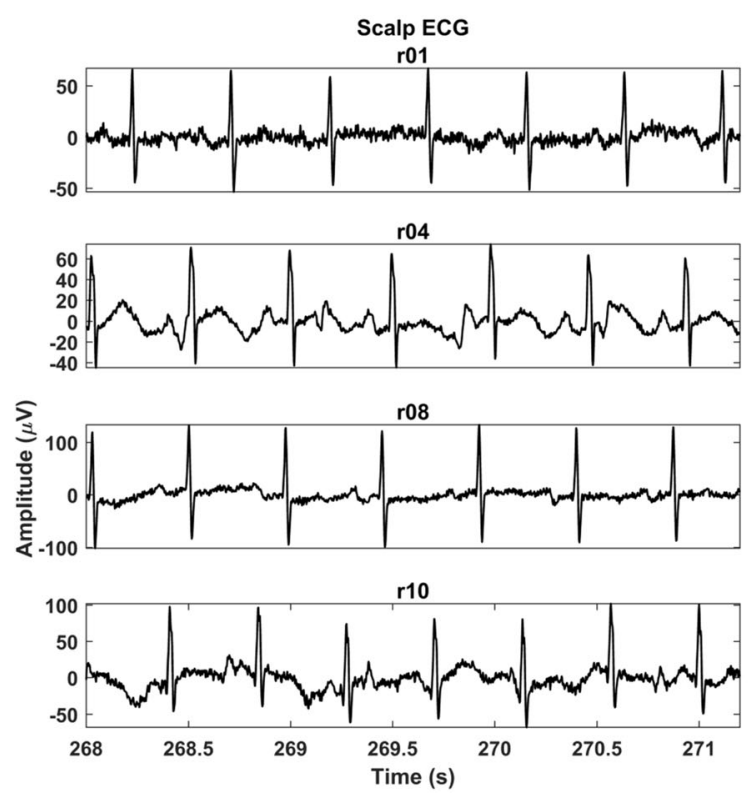

Fig. 9 The enhancement result of the proposed method (aTSAF) for the recordings "r01," "r04," "r08," and "r10" of the Abdominal and Direct Fetal Electrocardiogram Database. In the left column, the corresponding

very promising results. Second, in a practical application, the user could be informed to, in case of missing R peaks, distrust the output of the filter. Also, there is the possibility of using other measurement modalities to yield the regeneration times, like synchronous Doppler echocardiography as was proposed by Adam and Shavit [1].

Another shortcoming of the method is in cases of arrhythmia and ectopic beats. In these cases, sudden and brief changes are happening to ECG signal morphology and the filter is not able to adapt fast enough. As a consequence, it will only adapt to the morphology of regular beats and would be incapable of tracking the abrupt changes. This effect is also caused because of the averaging of ECG complexes for the enhancement of reference channels. Because of the averaging, brief variations in the ECG morphology are filtered out. However, without this enhancement step, the TSAF is unable to efficiently remove the noise. Moreover, the target application of this work is to enhance the fetal ECG quality for detecting hypoxia. The STAN method [33] that is used for fetal monitoring with an invasive electrode averages 30 consecutive heartbeats for ECG signal enhancement. For a typical fetal heart rate of about 140 beats per min (BPM), 30 heartbeats correspond to a time interval of $13 \mathrm{~s}$. Transient or structural changes in the ECG waveform that have clinical relevance with respect to developing hypoxia are hypothesized in the STAN methodology to occur over longer time scales. Consequently, averaging 30 consecutive ECG complexes preserves clinically relevant variations in the ECG and, at the same time, yields a substantial enhancement of signal quality. Based on the same reasoning, we also average 30 consecutive
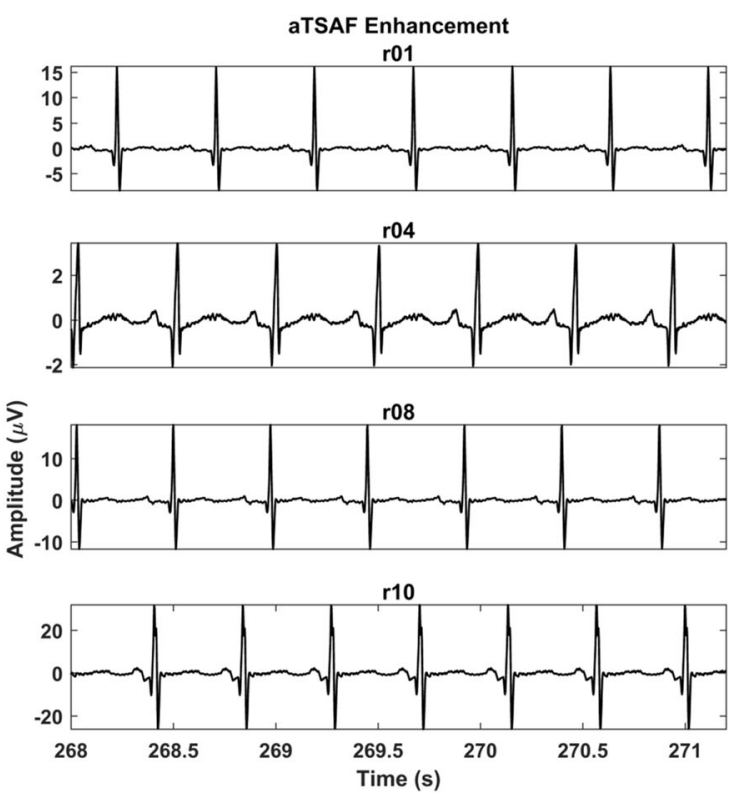

scalp ECGs are displayed. In all cases, the enhanced signals are clean and the morphological signal characteristics are similar to those of the scalp ECGs

ECG complexes to enhance our reference channels, while maintaining relevant information for detecting hypoxia.

The high-quality signals that the proposed method delivers can give the opportunity to clinicians to measure the exact timing of different morphological features of the ECG signal. Besides, it can facilitate and advance the research towards automated detection of fetal ECG intervals and segments. Extracting morphological features from the ECG signal allows for the estimation of the well-being of the fetus. Fetal acidosis is known to affect ECG morphology [17, 19], while asphyxia of the fetus is thought to be associated with changes in the $\mathrm{P}$ wave, PQ interval, and ST segment [29]. Moreover, fetal growth might also influence the timing of ECG waves [28].

\section{Conclusion}

This paper presented a method to improve the performance of the time-sequenced adaptive filter for fetal ECG enhancement. In the proposed method, the quality of the reference channels is enhanced prior to filtering via ensemble averaging of multiple consecutive ECG complexes. The evaluation of our filter, on both simulated and real fetal ECG signals, shows that the proposed algorithm outperforms the conventional timesequenced and the NLMS adaptive filtering techniques. Our results indicate that pre-processing of the reference channels provides a more accurate estimate of the underlying fetal ECG signal. The proposed algorithm can reveal the characteristic 
waves of the fetal ECG signal, even in cases with relatively low SNR. A limitation of our method is that, in case of rapidly changing ECG morphology, for instance in the presence of ectopic beats, our algorithm is unable to track these changes, yielding a suboptimal performance. Moreover, an estimate of the position of the fetal pulse locations is required to determine the regeneration times of the filter. Future work could focus on performing automated extraction of relevant morphological features such as PR intervals, QT intervals, and ST segments.

\section{Compliance with ethical standards}

Conflict of interest Eleni Fotiadou is funded by EU Horizon 2020 (grant number DLV-719500). Rik Vullings has shares in Nemo Healthcare BV, The Netherlands.

Open Access This article is distributed under the terms of the Creative Commons Attribution 4.0 International License (http://creativecommons. org/licenses/by/4.0/), which permits unrestricted use, distribution, and reproduction in any medium, provided you give appropriate credit to the original author(s) and the source, provide a link to the Creative Commons license, and indicate if changes were made.

\section{References}

1. Adam D, Shavit D (1990) Complete foetal ECG morphology recording by synchronized adaptive filtration. Med Biol Eng Comput 28:287-292

2. Andreotti F, Behar J, Zaunseder S, Oster J, Clifford GD (2016) An open-source framework for stress-testing non-invasive foetal ECG extraction algorithms. Physiol Meas 37(5):627-648

3. Andreotti F, et al. (2013) Maternal signal estimation by Kalman filtering and template adaptation for fetal heart rate extraction. Computing in Cardiology 2013. Zaragosa: IEEE. 193-196

4. Andreotti F, Riedl M, Himmelsbach T, Wedekind D, Wessel N, Stepan H, Schmieder C, Jank A, Malberg H, Zaunseder S (2014) Robust fetal ECG extraction and detection from abdominal leads. Physiol Meas 35(8):1551-1567

5. Behar J, Oster J, Clifford GD (2013) Non-invasive FECG extraction from a set of abdominal sensors. Computing in Cardiology 2013. Zaragosa: IEEE. 297-300

6. Camargo-Olivares JL, Martin-Clemente R, Hornillo-Mellado S, Elena MM, Roman I (2011) The maternal abdominal ECG as input to MICA in the fetal ECG extraction problem. IEEE Signal Process Lett 18(3):161-164

7. Camps G, Martinez M, Soria E (2001) Fetal ECG extraction using an FIR neural network. Comput Cardiol 28:249-252

8. Cano GG, Briller SA, Coast DA (1990) Enhancement of low-level ECG components in noise with time-sequenced adaptive filtering. J Electrocardiol 23:176-183

9. Clifford GD, Silva I, Behar J, Moody GB (2014) Non-invasive fetal ECG analysis. Physiol Meas 35:1521-1536

10. Douglas SC, Meng THY (1994) Normalized data nonlinearities for LMS adaptation. IEEE Trans Signal Process 42(6):1352-1365

11. Ferrara E, Widrow B (1981) Multichannel adaptive filtering for signal enhancement. IEEE Trans Acoust Speech Signal Process 29(3):766-770

12. Ferrara E, Widrow B (1981) The time-sequenced adaptive filter. IEEE Trans Acoust Speech Signal Process 29(3):679-683
13. Ferrara ER (1977) The time-sequenced adaptive filter. Stanford, CA: Ph.D. dissertation, Stanford University

14. Ferrara ER, Widrow B (1982) Fetal electrocardiogram enhancement by time-sequenced adaptive filtering. IEEE Trans Biomed Eng BME-29(6):458-460

15. Gao P, Chang E-C, Wyse L (2003) Blind separation of fetal ECG from single mixture using SVD and ICA. Fourth International Conference on Information, Communications and Signal Processing, 2003 and the Fourth Pacific Rim Conference on Multimedia. Proceedings of the 2003 Joint. Singapore: Information, Communications and Signal Processing, 2003 and 4rth Pacific Rim Conf. on Multimedia. Proc. of the 2003 Joint Conf. of the 4rth Int. Conf. on. 1418-22

16. Goldberger AL, Amaral LAN, Glass L, Hausdorff JM, Ivanov PC, Mar RG, Mietus JE, Moody GB, Peng C-K, Stanley HE (2000) PhysioBank, PhysioToolkit, and PhysioNet: components of a new research resource for complex physiologic signals. Circulation 101 (23): e215-e220

17. Greene KR, Dawes GS, Lilja H, Rosén K (1982) Changes in the ST waveform of the fetal lamb electrocardiogram with hypoxemia. Am J Obstet Gynecol 144:950-958

18. Hassanpour H, Parsaei A (2006) Fetal ECG extraction using wavelet transform. 2006 International Conference on Computational Inteligence for Modelling Control and Automation and International Conference on Intelligent Agents Web Technologies and International Commerce (CIMCA'06). Sydney: Computational Intelligence for Modelling, Control and Automation, 2006 and Int. Conf. on Intelligent Agents, Web Technologies and Internet Commerce, Int. Conf. on. 179-179

19. Jenkins HM, Symonds EM, Kirk DL, Smith PR (1986) Can fetal electrocardiography improve the prediction of intrapartum fetal acidosis? Br J Obstet Gynaecol 93:6-12

20. Jezewski J, Matonia A, Kupka T, Roj D, Czabanski R (2012) Determination of fetal heart rate from abdominal signals: evaluation of beat-to-beat accuracy in relation to the direct fetal electrocardiogram. Biomed Eng 57(5):383-394

21. Jia W, Yang C, Zhong G, Zhou M, Wu S (2010) Fetal ECG extraction based on adaptive linear neural network. 2010 3rd International Conference on Biomedical Engineering and Informatics. Yantai, China: 2010 3rd Int. Conf. on Biomedical Engineering and Informatics. 899-902

22. Kanjilal PP, Palit S, Saha G (1997) Fetal ECG extraction from single-channel maternal ECG using singular value decomposition. IEEE Trans Biomed Eng 44(1):51-59

23. Khamene A, Negahdaripour S (2000) A new method for the extraction of fetal ECG from the composite abdominal signal. IEEE Trans Biomed Eng 47(4):507-516

24. Laar van JO, Warmerdam GJ, Verdurmen KM, Vullings R, Peters $\mathrm{CH}$, Houterman S, Wijn PF, Andriessen P, van Pul C, Oei GS (2014) Fetal heart rate variability during pregnancy, obtained from non-invasive electrocardiogram recordings. Acta Obstet Gynecol Scand 93(1):93-101

25. Lindecrantz K (1983) Processing of the fetal ECG; an implementation of a dedicated real time microprocessor system. Chalmers University of Technology, Gothenburg

26. Martín-Clemente R, Camargo-Olivares JL, Hornillo-Mellado S, Elena M, Roman I (2002) Fast technique for noninvasive fetal ECG extraction. IEEE Trans Biomed Eng 58(2):227-230

27. Niknazar M, Rivet B, Jutten C (2013) Fetal ECG extraction by extended state kalman filtering based on single-channel recordings. IEEE Trans Biomed Eng 60(5):1345-1352

28. Pardi G, Ferrazzi E, Cetin I, Rampello S, Baselli G, Cerutti S, Civardi S (1986) The clinical relevance of the abdominal fetal electrocardiogram. J Perinat Med 14:371-377 
29. Pardi G, Tucci E, Uderzo A, Zanini D (1974) Fetal electrocardiogram changes in relation to fetal heart rate patterns during labor. Am J Obstet Gynecol 118(2):243-250

30. Podziemski P, Gieraltowski J (2013) Fetal heart rate discovery: algorithm for detection of fetal heart rate from noisy, noninvasive fetal ECG recordings. Computing in Cardiology 2013. Zaragosa: IEEE 333-336

31. Ramli RM, Noor AOA, Samad SA (2012) A review of adaptive line enhancers for noise cancellation. Aust J Basic \& Appl Sci 6(6):337-352

32. Rooijakkers JM, Rabotti C, Oei SG, Mischi M (2012) Lowcomplexity R-peak detection for ambulatory fetal monitoring. Physiol Meas 33(7):1135-1150

33. Rosén KG, Lindecrantz K (1989) STAN-the Gothenburg model for fetal surveillance during labour by ST analysis of the fetal electrocardiogram. Clin Phys Physiol Meas 10(4B):51-56

34. Sardy S (2000) Minimax threshold for denoising complex signals with Waveshrink. IEEE Trans Signal Process 48(4):1023-1028

35. Silva I, Behar J, Sameni R, Zhu T, Oster J, Clifford GD, Moody GB (2013) Noninvasive fetal ECG: the PhysioNet/Computing in Cardiology Challenge 2013. Comput Cardiol 40:149-152

36. Varanini M, Tartarisco G, Billeci L, Macerata A, Pioggia G, Balocchi R (2013) A multi-step approach for non-invasive fetal ECG analysis. Computing in Cardiology 2013. Zaragosa: IEEE. 281-284

37. Verdurmen KMJ, Lempersz C, Vullings R, Schroer C, Delhaas T, van Laar JOEH, Oei SG (2016) Normal ranges for fetal electrocardiogram values for the healthy fetus of 18-24 weeks of gestation: a prospective cohort study. BMC Pregnancy Childbirth 16(1):227

38. Vigneron V, Paraschiv-Ionescu A, Azancot A, Sibony O, Jutten C (2003) Fetal electrocardiogram extraction based on non-stationary ICA and wavelet denoising. Seventh International Symposium on Signal Processing and Its Applications, 2003. Proceedings. Paris, France: 7th Int Symp on Signal Processing and Its Applications, 2003. Proc. 69-72

39. Vullings R, de Vries B, Bergmans JWM (2011) An adaptive Kalman filter for ECG signal enhancement. IEEE Trans Biomed Eng 58(4):1094-1103

40. Widrow B, Glover JR, McCool JM, Kaunitz J, Williams CS, Hearn RH, Zeidler JR, Jr ED, Goodlin RC (1975) Adaptive noise cancelling: principles and applications. Proc IEEE 63(12):1692-1716
41. Wu S, Shen Y, Zhou Z, Lin L, Zeng Y, Gao X (2013) Research of fetal ECG extraction using wavelet analysis and adaptive filtering. Comput Biol Med 43(10):1622-1627

42. Ye Y, Sheu J, Zeng PCY, Wang G, Lu K (2009) An efficient semi-blind source extraction algorithm and its applications to biomedical signal extraction. Sci China Ser F-Inf Sci 52(10): 1863-1874

43. Zhang H, Shi Z, Guo C, Feng E (2009) Semi-blind source extraction algorithm for fetal electrocardiogram based on generalized autocorrelations and reference signals. J Comput Appl Math 223(1): $409-420$

Eleni Fotiadou is a current Ph.D. student in the Department of Electrical Engineering at the Technical University of Eindhoven (TU/e), The Netherlands, focusing on processing and analysis of non-invasive fetal electrocardiogram. She has received her Electrical and Computer Engineering diploma from the Aristotle University of Thessaloniki, Greece, in 2009

Judith van Laar is a maternal fetal medicine specialist in the Máxima Medical Center in Veldhoven, The Netherlands. She did a Ph.D. (dissertation in 2012), focusing on interpretation of fetal electrocardiogram recordings for fetal monitoring during pregnancy and labor, in the Department of Electrical Engineering at the Technical University of Eindhoven (TU/e), The Netherlands.

S. Guid Oei is currently a gynecologist-perinatologist in the Máxima Medical Center (MMC), Veldhoven, The Netherlands. He is also a professor of fundamental perinatology in the Department of Electrical Engineering at the Eindhoven University of Technology, The Netherlands. He received the Ph.D. degree from Leiden University, The Netherlands, in 1996.

Rik Vullings is an assistant professor in the Signal Processing Systems Group, Department of Electrical Engineering, Eindhoven University of Technology (TU/e), The Netherlands. His research focuses on robust, model-based analysis of biomedical signals. Next to his affiliation at TU/e, he is a chief scientific officer, co-founder, and shareholder of Nemo Healthcare BV, The Netherlands. 